\title{
Antioxidant synergism between ethanolic Centella asiatica extracts and $\alpha$-tocopherol in model systems.
}

\begin{abstract}
The synergistic antioxidant effects of ethanolic extracts of Centella asiatica (CE), and $\alpha-$ tocopherol have been studied. The types of interactions exhibited by $\mathrm{CE}$ and $\alpha$-tocopherol combined at different ratios were measured using three assays: 2,2'-azino-bis(3ethylbenzothiazoline-6-sulphonic acid) diammonium salt (ABTS) radical-scavenging capacity, the $\beta$-carotene bleaching system and liposome peroxidation assays. Fixed-fraction isobolographic analysis was used to detect any inducement of the antioxidant activity compared with the individual activities of $\mathrm{CE}$ and $\alpha$-tocopherol. Of all synergistic combinations of $\mathrm{CE}$ and $\alpha$-tocopherol, only fraction $2 / 3$ showed the synergistic combination that fits well in three different assays and can be explained by the regeneration of $\alpha$ tocopherol by $\mathrm{CE}$ despite the interaction effect of $\beta$-carotene present in the analytical assay. This phenomenon involved complex interactions between $\mathrm{CE}$ and $\alpha$-tocopherol to exhibit different degrees of interactions that eventually increased antioxidant activity.
\end{abstract}

Keyword: Synergism; Ultrasound; a-Tocopherol; Isobolographic analysis. 